\title{
Determinantes para a excelência na Orientação: as representações de treinadores e atletas de elite Determinants for excellence in Orienteering: the representation of elite coaches and athletes Determinantes para la excelencia en la orientación: las representaciones de entrenadores y atletas de elite \\ Tadeu Ferreira de Sousa Celestino, *José Carlos Gomes de Carvalho Leitão, **Antonino Manuel deAlmeida Pereira *University of Trás-os-Montes e Alto Douro (Portugal), ** Polytechnic Institute of Viseu (Portugal)
}

Resumo. A compreensão da excelência desportiva tem, desde sempre, proporcionado um intenso debate sobre os fatores que lhe estão subjacentes. Porém, no desporto orientação, a investigação no contexto da excelência tem sido escassa. Este estudo procurou identificar os fatores determinantes na aquisição, desenvolvimento e manutenção da excelência na orientação. Desenvolvido sob o paradigma qualitativo de análise, aplicou-se entrevistas semiestruturadas a um grupo de dez treinadores ibéricos de elite e a um grupo de catorze atletas de elite que integram as seleções nacionais da Suíça, Noruega, Dinamarca, França, Itália, Bulgária, Roménia e Letónia. Os resultados identificam que, similarmente, treinadores e atletas consideram os fatores secundários associados aos aspetos socio contextuais como a família, os amigos/pares e o treinador, e os aspetos socioculturais como os principais determinantes modeladores da excelência na orientação. Por outro lado, reconhecem que entre os fatores primários, os fatores psicológicos e da preparação desportiva se configuram como as principais variáveis de influência direta na performance de excelência. Salientam, igualmente, a necessidade da interação entre os diversos fatores para a aquisição, desenvolvimento e manutenção da excelência na orientação. Com a agregação destes resultados conclui-se que a excelência na orientação apresenta uma matriz multidimensional e a sua efetiva concretização é determinada pela interação bem-sucedida entre as dimensões do desempenho e as dimensões socio-contextuais e culturais que envolvem a modalidade orientação. Palavras-chaves: Expertise, treinador, desenvolvimento de atletas; excelência desportiva.

\begin{abstract}
The understanding of sport excellence has always provided an intense debate about the underlying factors. However, in sport orienteering, research in the field of excellence has been scarce. This study sought to identify the determining factors in the acquisition, development, and maintenance of excellence in orienteering. Developed under the qualitative analysis, semi-structured interviews were applied to a group of ten elite Iberian coaches and a group of fourteen elite athletes from the national teams of Switzerland, Norway, Denmark, France, Italy, Bulgaria, Romania, and Latvia. The results identify that, similarly, coaches and athletes consider secondary factors associated with socio-contextual aspects, such as family, friends / peers and coaches, and sociocultural aspects as the major determinants determining orienteering excellence. On the other hand, they recognize that among the primary factors, psychological factors and sports preparation are the main variables that directly influence performance of excellence. They also stress the need for interaction between the various factors for the acquisition, development, and maintenance of excellence in orienteering. Supported by these results, we may conclude that excellence in orientation presents a multidimensional matrix and its effective implementation is determined by the successful interaction between the dimensions of performance and the socio-contextual and cultural dimensions that involve the orienteering modality.
\end{abstract}

Keywords: Expertise, coach, developing athletes, sport excellence.

Resumen. La comprensión de la excelencia deportiva siempre ha proporcionado un intenso debate sobre los factores que le subyacen. Pero en el deporte orientacion, la investigación en el contexto de la excelencia ha sido escasa. Este estudio buscó identificar los factores determinantes en la adquisición, desarrollo y mantenimiento de la excelencia en la orientación. Desarrollado bajo el paradigma cualitativo de análisis, se aplicaron entrevistas semiestructuradas a un grupo de diez entrenadores ibéricos de élite ya un grupo de catorce atletas de elite que integran las selecciones nacionales de Suiza, Noruega, Dinamarca, Francia, Italia, Bulgaria, Rumanía y Letonia. Los resultados identifican que, de manera similar, entrenadores y atletas consideran los factores secundarios asociados a los aspectos socio contextuales como la familia, los amigos / parejas y el entrenador, y los aspectos socioculturales como los principales determinantes modeladores de la excelencia en la orientación. Por otro lado, reconocen que entre los factores primarios, los factores psicológicos y de la preparación deportiva se configuran como las principales variables de influencia directa en la performance de excelencia. También subrayan la necesidad de la interacción entre los diversos factores para la adquisición, el desarrollo y el mantenimiento de la excelencia en la orientación. Con la agregación de estos resultados se concluye que la excelencia en la orientación presenta una matriz multidimensional y su efectiva concreción está determinada por la interacción exitosa entre las dimensiones del desempeño y las dimensiones socio-contextuales y culturales que involucran la orientación.

Palabras claves: Expertise, entrenador, desarrollo de atletas, excelencia deportiva.

\section{Introdução}

A evolução a que assistimos no desporto tem transformado os seus conceitos, práticas emodelos de organização. Peseembora este progresso, o objetivo da excelência desportiva tem remanescido comometaaalcançar por uma grande maioria de indivíduos. No entanto, o que efetivamente se constata é que apenas uma pequena parte destes indivíduos consegue alcançar e manter níveis e performances de excelência (Gagné, 2007). Esta singularidade tem sido alvo de grande curiosidade e investigação por parte das Ciências do Desporto. Neste sentido as linhas de pesquisa desenvolvidas procuram compreender, essencialmente, que características e competências apresentam estes indivíduos? Como as desenvolveram e as mantém ao longo do tempo? Que fatores lhes estão subjacentes quelhes possibilita desenvolver a sua excecionalidade? Apesar

Fecha recepción: 07-05-17. Fecha de aceptación: 26-06-18 Tadeu Ferreira de Sousa Celestino titta2323@hotmail.com da diversidade e multidisciplinariedade de pesquisas alusivas ao tema, constata-se que a excelência desportiva apresenta, ainda, muitas questões por resolver (Leite, Santos, \& Sampaio, 2012). Desde logo a sua concetualizaçãoéfrequentemente conectada com termos como expertise, eminence, expert performance, alto rendimento, elite, talento entre outros (Matos, Cruz, \& Almeida, 2011). Em todos identifica-se um denominador comum que é a qualidade de ser superior comparativamente à generalidade dos indivíduos e, particularmente, em relação aos seus pares. Por outro lado, as diversas abordagens de estudo deste fenómeno humano indicam a existência de uma interação constante entre um conjunto de fatores de natureza interna e externa ao indivíduo que modelam a excelência (Renzulli, 2002; Gagné, 2007; Ericsson, Krampe, \& TeschRömer, 1993; Baltes, 1993) como sejam os aspetos genéticos, os relacionados com o treino e os aspetos psicossociais (Arias, 2008).

Noutro campo, observa-se também que os estudos desenvolvidos nas últimas décadas têm seguido uma tendência monodisciplinar, centrada, exclusivamente, nas perspetivas genetistas ou ambientalista como explicação da excelência (Philips, Davids, Renshaw, \& Portus, 2010). Por outras palavras, a investigação em torno da performance de excelência 
encontra-se dividida entre os estudos que sustentam a excecionalidade desportiva como consequência dos condicionalismos genéticos (Davids \& Baker, 2007), e aqueles que asseguram ser fundamentalmente oresultado da ação do contexto do desportista (Bloom, 1985; Côté, 1999; Ericsson et al., 1993). Porém, esta dicotomia entre nature vs nurture vem acentuar na atualidade a necessidade de uma visão mais eclética para a compreensão da interação e emergénese do talento excecional.

Neste sentido, e considerando os contributos de ambas perspetivas, Baker e Horton (2004) propuseram organizá-los e fazer uma distinção entre estes fatores. Assim, num primeiro grupo surgem os fatores primários, aqueles que exercem uma influência direta na performance, os quais incluem todos os elementos do próprio desportista agrupados em: 1) fatores psicológicos; 2) fatores genéticos e3) fatores da preparação desportiva (Baker \& Horton, 2004). Num segundo nível de influência surgem os fatores secundários, aqueles que exercem uma ação secundária e mediadora na relação entre as influências primárias e a performance desportiva (Baker \& Horton, 2004; Leite et al., 2012), como sejam os fatores contextuais, as características do treino, a influência do treinador ou da família, entre outros (Simón, Bustos \& Jordán, 2017).

Porém, constata-se que nem a combinação destes fatores é garantia de sucesso (Baker \& Horton, 2004; Arias, 2008), verificando-se, deste modo, que ainda se encontra por esclarecer qual a verdadeira magnitude e influência de cada um deles para um desempenho de excelência (Leite et al., 2012). Assim, mais recentemente, diversos autores têm vindo a argumentar que os desempenhos de excelência são uma consequência da interação bem-sucedida entre os fatores de ordem genética e ambiental do indivíduo (Baker \& Horton, 2004; Phillips et al., 2010), confirmando, assim, a necessidade de uma perspetiva mais holística para a compreensão deste fenómeno humano.

Apesar da relevância destas evidências, constatamos, particularmente no desporto de orientação, que o estudo da excelência tem seguidouma tendência unidimensional, centrada unicamentena análise isolada de um conjunto específico de variáveis associadas à performance de excelência, como seja o estudo: i) dos aspetos físicos (fisiológicos), aliados à caracterização morfológica dos atletas, na análise das variáveis e parâmetros fisiológicos como capacidade aeróbia, potência anaeróbia, resistência muscular e força muscular; ii) dos aspetos psicológicos, associados à compreensão dos processos cognitivos envolvidos na orientação, assim como no desenvolvimento e análise de estratégias para o controlo da autorregulação, como por exemplo o controlo da ansiedade, o pensamento positivo e a autoconfiança imagética, as técnicas de visualização do mapa, a motivação, atenção seletiva e a concentração; e iii) da interação entre os aspetos psicológicos e físicos (Celestino, Leitão, Sarmento, Routen \& Pereira, 2015).

Deste modo, ficam ainda por esclarecer algumas determinantes da performance igualmente importantes no processo de obtenção da excelência desportiva (Calleja \& Lorenzo, 2010; Lorenzo \& Sampaio, 2005), como sejam as variáveis ambientais, psicossociais e socioculturais associadas à orientação (Seiler, 1994). Efetivamente identifica-se uma escassez de pesquisas realizadas neste campo de estudo, e particularmente, sob um enfoque multidimensional eholístico, abordado sob uma perspetiva construtiva de análise. Assim, têm ficado por compreender os fatores, características e valores que os seus intervenientes diretos consideram estar subjacentes à excelência na orientação.

Face ao exposto, o objetivo deste estudo é o de conhecer os fatores que, segundo os treinadores (ex-selecionadores nacionais) e atletas de elite de orientação, são determinantes na aquisição, desenvolvimento e manutenção da excelência nesta modalidade desportiva.

\section{Método}

\section{Participantes dapesquisa}

A amostra foi constituída por dez treinadores ibéricos de orientação do sexo masculino, com uma média de idades de 42,7 $\pm 6,5$ anos, 9,6 \pm 2,9 anos de experiência como treinador, eleitos por terem sido selecionadores nacionais e terem acompanhado atletas em campeona- tos do mundo, e por um grupo de quinze atletas de elite de orientação, onze do sexo masculino e quatro do sexo feminino, com uma média de idades de 27,3 $\pm 3,6$, escolhidos por estarem integrados nas suas respetivas seleções de seus países (Suíça $n=4$; Noruega $=2$; Dinamarca $n=2$ França $n=2$; Itália $n=2$; Bulgária $n=1$; Roménia n =1; Letónia $\mathrm{n}=1$ ) e terem participado por mais que uma vez em provas do campeonato do mundo.

\section{Instrumentos}

A metodologia utlizada para a recolha dos dados foi a entrevista semiestruturada (Ghliglione \& Matalon, 2001). Os guiões da entrevista foram obtidos após os seguintes passos: i) preparação de um primeiro esboço com base nos objetivos do estudo e da literatura relevante no âmbito da excelência (Côté, Ericsson \& Law, 2005; Holt \& Dunn, 2004); avaliação do primeiro esboço de guião por três pesquisadores experts em Ciências do Desporto especialistas em metodologia qualitativa; iii) discussão dos resultados com base nas sugestões apresentadas por cada um dos especialistas; iv) realização de uma entrevista piloto com três elementos do universo em estudo; v) eventual reajuste em resultado da reflexão em grupo do estudo piloto. Finalizadas estas etapas, e sem alterações estruturais ao esboço inicial e com a unanimidade de concordância por parte dos especialistas, elaborou-se a versão final dos guiões que se encontram estruturados em três grandes dimensões de análise: i) o conceito de excelência-na qual se apresentam um conjunto de questões com o objetivo de aferir e compreender quais as conceções dos entrevistados acerca da excelência desportiva; ii) fatores que a influenciam - onde se apresentam um grupo de perguntas que procuram explorar o tipos de recursos subjacentes ao processo de formação desportiva, programas de deteção e desenvolvimento de talentos, pessoas significativas, procedimentos, habilidades que, sob o ponte de vista do entrevistado estão subjacentes à excecionalidade desportiva, e; iii) desenvolvimento emanutenção de excelência-na qual se apresentam um grupo de questões que procuram aferir as práticas, programas de treino, metodologias e estratégias de trabalho, no sentido de se compreender a evolução das performances excecionais no contexto da orientação.

\section{Procedimentos}

As entrevistas foram realizadas pelo autor entre 31 de julho de 2013 e 31 de março de 2015. Iniciaram-se com uma breve informação acerca dos propósitos do estudo. Seguidamente recolheram-se algumas informações demográficas, e por fim houve a exploração aprofundada do tema. Durante toda a entrevista os entrevistados tiveram tempo livre para responder as questões e esclarecer e reformular o seu pensamento. Cada entrevista teve entre 30 minutos e 1 hora de duração e com o consentimento dos entrevistados foi gravada em áudio e transcrita na íntegra.

A análise dos dados foi realizada por meio de análise de conteúdo (Bardin, 2008), recorrendo-se ao software QSR NVivo10 para a codificação das transcrições das entrevistas. A construção do sistema categorial foi realizada a priori e a posteriori (Bardin, 2008) sendo que as categorias resultantes foram submetidas ao painel de peritos, a fim de se cumprir as normas de fidelidade e validade de todo o processo.

\section{Resultados e Discussão}

Das entrevistas concedidas por este grupo de treinadores e atletas emergem um conjunto de fatores primários e secundários valorizados por este grupo de indivíduos por desempenharem um papel preponderante para a obtenção da excelência na orientação, como se observa na tabela 1 e 2 .

Porém, da análise dos seus discursos é possível identificar algumas similitudes e diferenças no que toca à valorização e contributo que cada um dos fatores tem na modelação da excelência na orientação. De seguida apresentamos, no âmbito dos fatores secundários, as similitudes e diferenças mais representativas apuradas. 


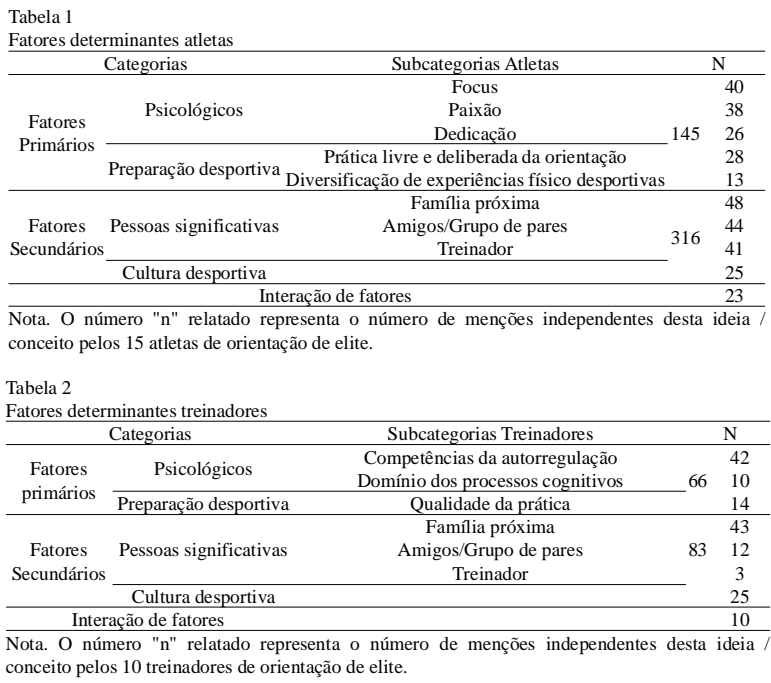

\section{Fatores Secundários}

Similitudes

De entre os fatores identificados, similarmente numa primeira instância, os fatores secundários (Baker \& Horton, 2004) são aqueles que, segundo a perceção dos nossos entrevistados, têm um maior grau de relevância quando se aborda a temática da excelência na orientação, tal como se observa:

«Acho que a maioria da minha excelência está relacionada com os aspetos culturais e sociais. Creio que muitas pessoas tem a motivação para ser melhores, quando têm um bom envolvimento.» Atleta 1 da seleção dinamarquesa

Com efeito, de entre estes fatores valorizados de modo similar por técnicos e atletas, reconhece-se a importância conferida às pessoas significativas (família próxima, amigos/pares e treinador) e aos aspetos socioculturais como importantes modeladores da excelênciana orientação. De entre estes, a família próxima assume uma especial relevância no processo para a excelência, uma vez que se configura como um dos principais catalisadores e potenciadores para a prática da modalidade e o desenvolvimento da excelência na modalidade, tal como se atesta:

«A orientação éde facto um desporto de família, não só pela questão de toda a família poder participar, que élogo uma mais-valia muito grande, mas principalmente porque um jovem que olha para os pais e os vêcorrer, vê-os envolvido, obviamente tem uma vantagem em termos de forma de estar que é extremamente importante para a excelência.» Treinador 6

«O suporte familiar é muito importante. Eu comecei muito cedo a

fazer orientação quando tinha 7 anos, porque o meu pai também fazia orientação.» Atleta 1 da seleção Suíça

Efetivamente, estudos desenvolvidos na modalidade (Celestino \& Pereira, 2012) evidenciam como uma das principais razões para a prática da orientação é esta poder ser acessível a todos. Deste modo é possível que num mesmo evento compitam e participem várias gerações familiares. Com efeito, a presença da família próxima nas fases iniciais de desenvolvimento, revela-se crucial para a aprendizagem e desenvolvimento dos skills básicos da orientação. No mesmo sentido, os familiares constituem-se importantes meios de suporte emocional, logístico, instrumental e económico, essenciais, quer no início quer para a continuidade da carreira desportiva e consequente desenvolvimento da excelência (Baker \& Horton, 2004; Bloom, 1985; Côté, 1999).

Igualmente relevante para os nossos entrevistados surge o papel determinante desempenhado pelos amigos/pares em consequência dos fortes laços que os une.

«São os amigos. Ter um bom grupo de atletas que se entreajudem, que puxem uns pelos outros, isso é muito importante. Ter uma equipa com fortes laços é uma das bases do sucesso. Atleta 2 da seleção Suíça

«É impensável um atleta de excelência devido a exigência da sua rotina, da sua vida conseguir fazer isso tudo se não tiver a concordância, o apoio e o incentivo dos seus pares, amigos e outros, mas fundamentalmente dos seus pares.» Treinador 7

Em consequência, esta valorização resulta no reconhecimento do apoio motivacional, promoção da alegria, satisfação com a prática e consequente incentivo na superação de obstáculos e continuidade na modalidade que as amizades proporcionam (Gould, Dieffenbach, \& Moffett, 2002). Deste modo, configura-se ser determinante para o desenvolvimento emanutenção da excelência na orientação, uma estrutura de suporte social com a criação de fortes laços de amizade, companheirismo entre pares, para a transmissão de saberes, experiências e superação de dificuldades, comprometimento e evolução nas performances (Baker \& Horton, 2004; Bloom, 1985).

Num campo mais vasto, os nossos entrevistados salientam o influente papel dos fatores socioculturais que envolvem a orientação, nomeadamente a cultura desportiva assumida em determinadas regiões do mundo. Com efeito, é nos países da região escandinava onde a orientação tem uma maior representatividade, disponibilizando uma infinidade de recursos instrumentais que favorecem quer o desenvolvimento da orientação, quer possibilitando o acesso a uma melhor formação e condições ímpares para a excelência como referido:

«Nos clubes da Escandinávia há possibilidade de treino para toda a gente. Acho que quando estás realmente na orientação e queres ser um bom orientista tens que ir para um país escandinavo. Mesmo nós, atletas suíços, temos de ir para lá desenvolver os nossos skills.» Atleta 3 da seleção Suíça

«O fator da importância que a modalidade tem em determinado país, porque a partir daí vêm todos os outros fatores. Por mais que os atletas tenham todas as características se não tiver condições de treino, se não tiver apoios ... torna-se impensável. «Treinador 7

«Eu decidi mudar-me para a Suécia onde o envolvimento ébom... há mais cultura da orientação. Lá (Escandinávia) tens mais atletas, mais competições, muito bons mapas, muito bons terrenos todas estas coisas que te tornam melhores.» Atleta da seleção búlgara

Efetivamente, uma performance de excelência na orientação requer a hábil conjugação entre as componentes físicas cognitivas (Sirin, Bektas \& Duman, 2011), onde o domínio perfeito dos processos cognitivos são variáveis imprescindíveis (Seiler, 1990). Deste modo, o domínio exímio da capacidade da deteção e uso da informação do mapa e do terreno não depende apenas da situação em si, mas principalmente, das experiências anteriores vivenciadas ao longo do tempo(Oliveira \& Duarte, 2005). Consequentemente, a experienciação através do usufruto de recursos de qualidade (mapas, terrenos, cartografia, treinadores) são elementos considerados preponderantes no processo de obtenção e manutenção da excelência. Com efeito, é na região escandinava que a orientação assume uma maior representatividade, expressão cultural e disponibilidade de recursos de elevado valor, que, de acordo com estes treinadores eatletas, configuram-se requisitos indispensáveis no processo de desenvolvimento da excelência na orientação. Por outro lado, o grau de importância que um determinando país, sociedade ou cultura atribui a uma determinada prática ou modalidade, tem uma influência preponderanteno seu desenvolvimento, manutenção e sucesso (Baker \& Horton, 2004; Lorenzo \& Sampaio, 2005) e consequentemente no desenvolvimento dos seus praticantes.

\section{Fatores Primários}

\section{Similitudes}

Noutra perspetiva, similarmente relevante para os entrevistados, identifica-se a consciencialização de que a efetiva aquisição, desenvolvimento e manutenção da excelência na orientação requer a interação bem-sucedidas entre os diversos fatores que se lhe encontram subjacentes, como se atesta nos seus discursos:

«Existem muitos parâmetros que têm de estar juntos como um puzzle da boa performance. Para ter uma boa performance é necessário o talento básico, depois necessitas de trabalhar com o envolvimento, como clubes, família... ter possibilidades de treino no local onde vives e isso é importante para se alcançar o topo ... tem que se encontrar muito equilíbrio em tudo.» Atleta da seleção 
romena

«As duas interligam-se, a genética já tem de ter e depois o meio que a desenvolve é bastante importante. Portanto, as coisas têm de estar ... forçosamente interligadas ... Essa interligaçãoé feita desde a formação onde se vai incutindo ao atleta todos estes fatores, ... e ele quando chega lá acima já os tem todos os fatores interligados.» Treinador 3

Com efeito, a especificidadeda orientação, onde se exige a interligação das componentes físicas e cognitivas para a obtenção de uma boa performance (Seiler, 1990), a sua multidisciplinariedade, os aspetos socioculturais que the são inerentes (Celestino \& Pereira, 2012), fundamentam a necessidade de uma interação entre fatores para a excelência na modalidade. No mesmo sentido, estas representações apuradas vão ao encontro das mais recentes perspetivas que procuram esclarecer o fenómeno da excelência. Estas sustentam a excelência como um fenómeno que se desenvolve ao longo do tempo, justamente através da interação bem-sucedida das características do atleta e do seu contexto, considerando-se inclusive, a base principal para a excelência desportiva (Davids \& Baker, 2007; Ericsson, Roring \& Nandagopal, 2007; Leite et al., 2012; Phillips et al., 2010).

\section{Fatores Primários}

\section{Diferenças}

Quanto aos fatores primários, os treinadores e atletas entrevistados consideram-nos igualmente importantes para a aquisição, desenvolvimento e manutenção da excelência na orientação. Entre eles, identificam-se os fatores psicológicos e da preparação desportiva como os mais valorados. Porém, reconhece-se algumas dissimilitudes quanto à importância que os técnicos e atletas lhe atribuem.

Ao nível dos fatores psicológicos, os treinadores valorizam o importante papel desempenhado pelas competências de autorregulação (como a concentração, autoconfiança, autocontrolo, autoestima, o pensamento positivo, a motivação), assim como a necessidade do domínio perfeito dos processos cognitivos associados à orientação (Seiler, 1990,1991), tal como se pode identificar nas suas falas:

«Todos esses tópicos psicológicos são mesmo muito importantes.

Num atleta de orientação é isso que o faz fazer provas a uma velocidade incrível, a navegar em terrenos desconhecidos a uma velocidade inacreditável mesmo sem nunca ter estado nesses terrenos. Issoéo mais impressionante. Sementalmente, psicologicamente, eu não tiver os meus pensamentos todos «alinhados», eu nunca vou conseguir estar a trabalhar a parte técnica e a parte tática da modalidade ... É imprescindível ter um nível de autoconfiança muito elevado porque, na orientação, estamos sempre a tomar decisões e eu tenho de ter confiança nas minhas decisões, elas terão de ser sempre as melhores do mundo.» Treinador 8

Esta perceção dos treinadores está relacionada com a necessidade dos atletas desenvolverem estas competências por forma a adquirem autonomia em competição. Por outras palavras, a especificidade da orientação, o contexto onde ela se desenvolve e caracter isolado com o qual o atleta toma decisões na resolução dos problemas com que se defronta, justificam a necessidade de desenvolver estas competências, consideradas por Seiler (1991) como um meio fundamental para se elevar e manter os níveis da performance na orientação. Neste sentido, o desenvolvimento destas competências possibilita posteriormente aos atletas, um ajustamento do seu estado emocional, auxiliando e facilitando a tomadas de decisão face aos problemas de navegação e reduzindo inequivocamente os erros de navegação. Também o desenvolvimento destas competências é reconhecido por diversos estudos (MacNamara, Button, \& Collins, 2010), os quais sublinham o seu valor, impacto ao nível da performance e o contributo inequívoco para o desenvolvimento da excelência desportiva.

Ainda no âmbito dos fatores psicológicos, a valorização do domínio dos processos cognitivos, foi também considerada pelos treinadores como preponderante e distintivo da performance de excelência na orientação, tal como um treinador refere:

«Um atleta tem de estar completamente confortável naquilo que está a fazer a cada segundo da sua performance. Para atingir a perfeição, para atingir um rendimento alto, ele tem de ser capaz de saber perfeitamente o porquê de em cada momento escolher cada uma das estratégias de navegação.» Treinador 7

Efetivamente, o sucesso das ações na orientação está intimamente relacionado com a forte carga cognitiva que envolve a componente estratégica e as técnicas de orientação. Deste modo, o domínio perfeito dos processos cognitivos configura-se um requisito imprescindível à performance de excelêncianamodalidade(Seiler, 1991). Por conseguinte, ler a informação do mapa, compará-la com o terreno real, comprovar as características do terreno e relocalizar-se, são processos cognitivos altamente exigentes que caracterizam a especificidade da modalidade(Seiler, 1990; 1991), constituindo o seu domínio uma componente peculiar da excelência na orientação(Seiler, 1990).

Por seu turno, ao nível dos fatores psicológicos, os atletas manifestam uma maior valorização dos mesmos associados ao focus, paixão e a dedicação ao desporto, e em especial à orientação, como se constata:

«Para mim é muito claro, a tua mente tem de estar focada se queres ser excelente na orientação.» Atleta 4 da seleção Suíça

«Eu acho que é uma paixão. Quando tu queres ser melhor tens que ter paixão por aquilo que fazes, paixão para vencer e para ser bom. Não é apenas uma questão de treino físico e técnico é algo mais relacionado com a paixão.» Atleta 3 da seleção Suíça

«Tu queres aperfeiçoar todos os aspetos da performance da orientação e para isso precisas de muita dedicação.» Atleta 3 da seleção Suíça

Estas perceções dos nossos entrevistados são consistentes com a literatura de referência, onde estes aspetos psicológicos se revelam importantes modeladores da excelência desportiva(Baker \& Horton, 2004; Gould et al., 2002). Com efeito, as exigências do treino, a especificidade técnica e tática da orientação e a própria meta de alcançar manter a excelência, exigem uma elevada capacidade de focus valorizada por estes atletas. O focus configura-se como uma das características fundamentais subjacentes à excelência, funcionando como um catalisador direcional para o cumprimento de metas (Orlick, 2008). No mesmo sentido, e estreitamente relacionado com o focus, surge a paixão, valorada por este grupo de atletas como o reflexo do envolvimento, entrega e persistência na superação de obstáculos para alcançar a excelência (Vallerand et al. 2008), bem como traduz o gosto e o prazer que sentem com a prática da modalidade. Consequentemente identifica-se a paixão como uma importante fonte de motivação, justificando toda a dedicação e empenho em querer ser melhor (Bloom, 1985, Vallerand, Salvy, Mageau, Elliot, Denis, Grouzet, \& Blanchard, 2007).

\section{Fatores Secundários}

\section{Diferenças}

Quanto aos fatores relacionados com a preparação desportiva, os técnicos entrevistados dão especial ênfase à qualidade da prática desenvolvida ao longo de um longo período de tempo nunca inferior a dez anos, tal como é referido por alguns:

«É muito raro aparecer um atleta que tem rendimento que não começou a fazer orientação logo aos 7, 8 anos, porque na orientação isto leva muito tempo, leva muitos anos a conseguir melhorar-se.» Treinador 7

«Tens que lhes ensinar qualidade, não somente pô-los a correr com o mapa ...» Treinador 9

«Tem que se ir fazendo o atleta de modo muito sustentado. Ele não nasce atleta, no mínimo necessário dez, doze anos.» Treinador 3

Esta perceção apurada reflete a necessidade que o desenvolvimento dos automatismos e skills associados a um alto rendimento na orientação exige um longo processo de consolidação, modelado por uma boa estruturação das competências a desenvolver ao longo deste processo. Destemodo, desenvolver e consolidar estas competências é um processo complexo e consequentemente longo (Baker, Côté \&Albernethy, 2003a; 2003b), o qual tem a sua génese durante a infância (Barreiros et al., 2013) e que se traduz numa intensa e adequada formação desportiva. 
Inerentemente a este processo, sobressai o compromisso deliberado, quer com a prática desportiva informal, quer formal (Baker et al., 2003a; Bloom, 1985; Ericsson et al., 1993), com efeitos cumulativos, uma vez que parece existir uma forte correlação positiva entre os anos acumulados de prática e o desempenho desportivo (Vllaroel, Mora \& GonzálezParra, 2011), e o consequente desenvolvimento da excelência desportiva (Baker et al., 2003a; 2003b).

Por seu turno, o grupo de atletas de elite entrevistados valoriza mais a experiência adquirida com a diversificação das experiências físico desportivas relacionadas com a prática de outras modalidades e com a prática livre e deliberada da orientação (diversificação de terrenos, cartografia,...), como sugerem:

«Na minha opinião tenho que admitir que algo muito importanteé a experiência adquirida com a passagem por muitos diversos tipos de terreno. Isto é fundamental para se alcançar a excelência na orientação ... não acho que correr, treinar apenas em mapas escandinavos, ou correr apenas com mapas italianos se possa alcançar a excelência épreciso correr sobre diversos tipos.» Atleta 1 da seleção italiana

«Com o desenvolvimento de um conjunto de imagens de diferentes tipos de terreno, vais conseguir estar melhor preparado para enfrentar e melhorar em qualquer tipo de situação. Se visitares muitos diferentes tipos de terrenos ficas preparado para tudo.» Atleta 4 da seleção Suíça

Assim, infere-se das narrativas dos atletas que um dos principais fatores para a excelência na orientação reside na necessidade da deliberada diversificação dos locais de treino, por forma a experienciar e contactar com diversas realidades de cartografia, mapas e terrenos. Considerando a componente física e cognitiva que envolve a orientação, a aquisição de um reportório diversificado de experiências eleva o conhecimento processual e declarativo e as adaptações de memória dos atletas. Consequentemente estas experiências possibilitam a codificação e recuperação de informações específicas de forma mais rápida e flexível durante o processo de navegação elevando a capacidade de antecipação e, deste modo, a eficiência e a eficácia da navegação (Eccles, Walsh \& Ingledew, 2002).

Em suma, o nível de competência, habilidade ou expertise no desempenho desportivo está intimamente relacionado com o aumento significativo da experiência. Com efeito, quer o conhecimento, quer a qualidade das habilidades aumentam consideravelmenteem consequência do elevar das situações de prática e competição (Iglesias, et al., 2010). Porém, a prática não se configura, por si só, como preditora da excelência. Como tal, deverá ser altamente estruturada, diferenciando-se do treino ou da experiência pela inclusão intencional de atividades desenvolvidas para a promoção do desempenho.

Em associação, nos discursos deste grupo de atletas, constata-se a importância que estes atribuem à prática de outras modalidades desportivas para além da orientação como um dos pré-requisitos da excelência quando referem:

«Recomendo fazer outros desportos, não focar-se apenas numa só coisa.» Atleta 2 da seleção Suíça

«Ter uma boa base de outros de outros desportos.» Atleta 1 da seleção francesa

«Para mim, acho não ser muito importante fazer só orientação quanto tens 10 anos. O mais importante é interessar-te pela prática desportiva diversificada não só orientação, mas também correr, jogar futebol, jogos desportivos coletivos, ter uma boa coordenação.» Atleta 4 da seleção Suíça

«Desenvolver uma boa dose de endurance, velocidade, flexibilidade, andar muito de bicicleta, ou cross country, ski no inverno, treinos alternativos, também é importante.» Atleta 2 da seleção Suíça

Efetivamente, ao longo do processo do seu desenvolvimento, os atletas vão vivenciando um conjunto de atividades físico-desportivas, que no seu conjunto promovem a aquisição de um reportório de skills preceptivo-motores que se repercutem, determinantemente, no aperfeiçoamento das capacidades físicas e cognitivas necessárias para a modalidade principal (Baker et al., 2003b). Esta perceção por nós apu- rada encontra-se em sintonia com os resultados de diferentes estudos que têm vindo a destacar a relevância da prática desportiva diversificada como um pré-requisito essencial da excelência desportiva (Baker et al., 2003a; Bloom, 1985). Num estudo desenvolvido com triatletas Baker, Deakin eCôté(2005) identificam que a expertise no triatlo está associada a um extenso comprometimento com uma diversidade de práticas desportivas.

\section{Conclusões}

Apesar do forte interesse que a temática da excelência tem no âmbito das ciências do desporto, são ainda escassos os estudos desenvolvidos sob uma perspetiva holística e integradora no âmbito da orientação. Neste sentido, o nosso estudo, partindo de uma perspetiva exploratória, procurou identificar os fatores mais representativos, que segundo treinadores e atletas de elite de orientação consideram desempenharum papel preponderante para a aquisição, desenvolvimento emanutenção da excelência nesta modalidade.

Distinguidos em fatores primários e secundários mediante a sua ação e influência ao nível da performance, os dados apurados permitemnos concluir que os fatores secundários relacionados com os aspetos socio-contextuais como a família, os amigos/pares, o treinador e a cultura desportiva, são aqueles que similarmente técnicos e atletas consideram ser determinantes modeladores no processo para o desenvolvimento da excelência na orientação.

Por outro lado, conclui-se que entre os fatores primários, os psicológicos e da preparação desportiva, se configuram como determinantes e de influência direta na performance de excelência na orientação. Não obstante, entre estes, os treinadores diferenciam e valorizam mais as competências de autorregulação e domínio dos processos cognitivos, assim como a qualidade da prática desenvolvida ao longo de um longo período de tempo. Por seu turno, os atletas valoram o focus, a paixão e a dedicação, bem como a diversificação das experiências físicodesportivas e a deliberada prática da orientação, como variáveis determinantes de influência direta na performance de excelência.

Conclui-se igualmente ser relevante a necessidade de haver uma interação bem-sucedida entre os diversos fatores modeladores da excelência neste contexto, o que vai ao encontro das mais recentes evidências que procuram compreender o fenómeno da excelência humana.

Os resultados obtidos permitem-nos concluir que a excelência na orientação se desenvolve ao longo do tempo com base na interação bemsucedida entre os diversos fatores que a influenciam. Assim, reconhecese a excelência desportiva como um fenómeno multidimensional identificando-se que na orientação, mais que as dimensões do desempenho, é importante reconhecer as dimensões socio-contextuais e culturais que envolvem os atletas.

Uma das limitações deste estudo prende-se, essencialmente, com a escassa pesquisa desenvolvida na modalidade relativa à análise dos fatores subjacentes à excelência, com a qual possamos confrontar os nossos resultados. Do mesmo modo, e face ao caráter exploratório do estudo, parece-nos também que seria pertinente a utilização de uma abordagem mista ao nível da investigação, integrando também a vertente quantitativa. Essa abordagem mista possibilitaria uma maior abrangência e profundidade, conferindo mais robustez aos resultados obtidos.

Apesar do estudo poder aportar alguns contributos para a compreensão do fenómeno da excelência desportiva na orientação, entendemos que mais pesquisas são necessárias para preencher o hiato existente na bibliografia ao nível desta temática.

Face ao exposto, esta pesquisa conforma um ponto de partida para o aprofundamento do estudo da excelência na orientação. Os dados apurados permitem-nos colocar algumas questões que merecem um maior aprofundamento e estudo, nomeadamente compreender: Qual a real contribuição dos diferentes fatores, particularmente os relacionados com dinâmicas contextuais e socioculturais, que afetam os atletas de orientação nas diversas etapas de desenvolvimento? Que atividades físicas desportivas se devem desenvolver para uma efetiva diversificação 
da preparação desportiva? Como e quando se deve processar a sua prática ao longo do percurso de desenvolvimento do atleta?

\section{Referências}

Arias Estero, J. L. (2008). El proceso de formación deportiva en la iniciación a los deportes colectivos fundamentado en las características del deportista experto. Retos. Nuevas tendencias en Educación Física, Deporte y Recreación,13, 28-32.

Baltes, P. B. (1993). The aging mind: potential and limits. Gerontologist, 33(5), 580-594. doi: 10.1093/geront/33.5.580

Baker, J., Côté, J., \& Abernethy, B. (2003a). Learning from the experts: practice activities of expert decision makers in sport. Research Quarterly for Exercise and Sport, 74(3), 342 347. doi: 10.1080/ 02701367.2003.10609101

Baker, J. Côté, J., \& Albernethy, B. (2003b). Sport-specific practice and the development of expert decision-making in team ball sports. Journal of Applied Sport Psychology, 15(1), 12-25. doi: 10.1080/ 10413200305400

Baker, J., Deakin, J., \& Côté, J. (2005). On the utility of deliberate practice: Predicting performance in ultra-endurance triathletes from training indices. International Journal of Sport Psychology, 36(3), 225-240.

Baker, J., \& Horton, S. (2004). A review of primary and secondary influences on sport expertise. High Ability Studies, 15(2), 211-228. doi:10.1080/1359813042000314781

Baker, J., Horton, S., Robertson-Wilson, J., \& Wall, M. (2003). Nurturing sport expertise: Factors influencing the development of the elite athlete. Journal of Sports Science and Medicine, 2(1), 1-9.

Bardin, L. (2008). Análise de conteúdo. Lisboa: Edições 70.

Barreiros, A. Côté, J., \& Fonseca,A.M, (2013). Sobre o desenvolvimento do talento no desporto: um contributo dos modelos teóricos do desenvolvimento desportivo. Revista de Psicologia del Deporte, 22(2), 489-494.

Bloom, B. (1985). Developing talent in young people. New York: Ballantine.

Calleja, J., \& Lorenzo, A. (2010). Factores condicionantes del desarrollo deportivo. Bizkaia: Diputación Foral de Bizkaia Dirección General de Deportes.

Celestino, T., \& Pereira, A. (2012). The sport of orienteering: representations of leisure sportive physical activity practitioners' without any experience on this modality. Cultura Ciência e Deporte, 7(19), 45-52. doi:10.12800/ccd.v7i19.24

Celestino, T., Leitão, J., Sarmento, H., Routen, A., \& Pereira,A. (2015). Elite Coaches views on factors contributing to excellence in orienteering. Cultura Ciência e Deporte, 10(28), 77-86. doi:10.12800/ccd.v10i28.517

Côté, J. (1999). The influence of the family in the development of talent in sport. The Sport Psychologist, 13(4), 395-417.

Côté, J., Ericsson, K.A., \& Law, M. P. (2005). Tracing the development of athletes using retrospective interview methods: A proposed interview and validation procedure for reported information. Journal of Applied Sport Psychology, 17(1), 1-19. doi: 10.1080/ 10413200590907531

Davids, K., \& Baker, J. (2007). Genes, environment and sport performance. Why the nature-nurture dualism is no longer relevant. Sports Medicine, 37(11), 961-980.

Eccles, D. W., Walsh, S. E., \& Ingledew, D. K. (2002). A grounded theory of expert cognition in orienteering. Journal of Sport and Exercise Psychology, 24(1), 68 -88.

Ericsson, K. A., Roring, R. W. \& Nandagopal, K. (2007). Misunderstandings, agreements, and disagreements: Toward a cumulative science of reproducibly superior aspects of giftedness. High Ability Studies, 18(1), 97-115. doi: 10.1080/ 13598130701351088

Ericsson, K. A., Krampe, R., \& Tesch-Römer, C. (1993). The role of deliberate practice in the acquisition of expert performance.
Psychological Review, 100(3), 363-406. doi:10.1037/0033295X.100.3.363

Gagné, F. (2007). Ten commandments for academic talent development. Gifted Child Quarterly, 51(2), 93-118.

Gould, D., Dieffenbach, K., \& Moffett, A. (2002). Psychological characteristics and their development in Olympic Champions. Journal of Applied Sport Psychology, 14(3), 172-204. doi: 10.1080/ 10413200290103482

Holt, N. L., \& Dunn, J. G. (2004). Toward a grounded theory of the psychosocial competencies and environmental conditions associated with soccer success. Journal of Applied Sport Psychology, 16(3), 199-219. doi: 10.1080/10413200490437949.

Iglesias Gallego, D., García González, L., García Calvo, T., Del Barco, B. L., \& del Villar Álvarez, F. (2010). Expertise development in sport: contributions under cognitive psychology perspective. Journal of Human Sport and Exercise, 5(3), 462-475. doi:10.4100/ jhse.2010.53.16.

Leite, N., Santos, \& Sampaio, J. (2012). Preparação Desportiva a Longo Prazo: uma abordagem multifatorial. Vila Real: Universidade de Trás-os-Montes e Alto Douro.

Lorenzo, A., \& Sampaio, J. (2005). Reflexiones sobre los factores que pueden condicionar el desarrollo de los deportistas de alto nivel. Aputs Educación Física e Deportes, 80, 63-70.

MacNamara, Á., Button, A., \& Collins, D. (2010). The role of psychological characteristics in facilitating the pathway to elite performance part 1: Identifying mental skills and behaviors. The Sport Psychologist, 24(1), 52-73.

Matos, D. S., Cruz, J. F., \& Almeida, L. S (2011). Excelência no desporto: Para uma compreensão da «arquitectura dos atletas de elite. Motricidade, 7(4), 27-41. doi: 10.6063/motricidade.7(4).

Phillips, E., Davids, K., Renshaw, I., \& Portus, M. (2010). Expert performance in sport and the dynamics of talent development. Sports Medicine, 40(4), 271-283. doi: 10.2165/11319430000000000-00000

Renzulli, J. S. (2002). Emerging conceptions of giftedness: Building a bridge to thenew century. Exceptionality, 10(2), 67-75. doi: 10.1207/ S15327035EX1002_2

Seiler, R. (1990). Decision Making Processing in Orienteering: an action theoretical investigation. International Journal of Sport Psychology, 21, 36-45.

Seiler, R. (1991). Psychological skills training in orienteering. Scientific Journal of Orienteering, 7, 74-85.

Seiler, R. (1994). Recent Trends and Future Directions of Research in Orienteering. Scientific Journal of Orienteering, 10(1/2), 3-23.

Simón Piqueras, J.A., Fernández Bustos, J. G., \& Contreras Jordán, O. R. (2017). Diseño y validación de un cuestionario de autopercepción de la excelencia en el deporte. Retos Nuevas Tendencias en Educación Física, Deporte y Recreación, 31, 58-63.

Sirin, E.F., Bektas, F. \& Duman, S. (2011). The relationship between orienteering athletes' multiple intelligence domains and their decision making styles. World Apllied Sciences Journal, 14(2), 228-234.

Oliveira, F., \& Duarte, A. (2005). A tomada de decisão na orientação. In D.F.Araújo(Ed.), O contexto da decisão: Acção táctica no desporto. (pp. 285-310). Lisboa: Visão e Contextos.

Orlick, T. (2008). In pursuit of excellence ( $4^{\mathrm{a}}$ Ed.). U.K. Human Kinetics.

Vallerand, R., Geneviève, M., Elliotb, A., Dumaisc, A., Demersd, A., \& Rousseaue, F. (2008). Passion and performance attainment in sport. Psychology of Sport and Exercise, 9(3), 373-392. doi: 10.1016/ j.psychsport.2007.05.003

Vallerand, R. J., Salvy, S. J., Mageau, G. A., Elliot, A. J., Denis, P. L., Grouzet, F. M., \& Blanchard, C. (2007). On the role of passion in performance. Journal of Personality, 75(3), 505-534. doi:10.1111/ j.1467-6494.2007.00447.x

Villaroel, C., Mora, R., \& González-Parra, G. C. (2011). Elite triathlete performance related to age. Journal of Human Sport and Exercise, 6(2), 363-373. doi:10.4100/jhse.2011.62.16 